\title{
Graphs with multiplicative vertex-coloring 2-edge-weightings
}

\author{
Joanna Skowronek-Kaziów ${ }^{1}$
}

Published online: 20 October 2015

(C) The Author(s) 2015. This article is published with open access at Springerlink.com

\begin{abstract}
A $k$-weighting $w$ of a graph is an assignment of an integer weight $w(e) \in$ $\{1, \ldots k\}$ to each edge $e$. Such an edge weighting induces a vertex coloring $c$ defined by $c(v)=\prod_{v \in e} w(e)$. A $k$-weighting of a graph $G$ is multiplicative vertex-coloring if the induced coloring $c$ is proper, i.e., $c(u) \neq c(v)$ for any edge $u v \in E(G)$. This paper studies the parameter $\mu(G)$, which is the minimum $k$ for which $G$ has a multiplicative vertex-coloring $k$-weighting. Chang, $\mathrm{Lu}, \mathrm{Wu}, \mathrm{Yu}$ investigated graphs with additive vertex-coloring 2-weightings (they considered sums instead of products of incident edge weights). In particular, they proved that 3-connected bipartite graphs, bipartite graphs with the minimum degree 1, and $r$-regular bipartite graphs with $r \geq 3$ permit an additive vertex-coloring 2 -weighting. In this paper, the multiplicative version of the problem is considered. It was shown in Skowronek-Kaziów (Inf Process Lett 112:191-194, 2012) that $\mu(G) \leq 4$ for every graph $G$. It was also proved that every 3-colorable graph admits a multiplicative vertex-coloring 3 -weighting. A natural problem to consider is whether every 2-colorable graph (i.e., a bipartite graph) has a multiplicative vertex-coloring 2-weighting. But the answer is no, since the cycle $C_{6}$ and the path $P_{6}$ do not admit a multiplicative vertex-coloring 2-weighting. The paper presents several classes of 2-colorable graphs for which $\mu(G)=2$, including trees with at least two adjacent leaf edges, bipartite graphs with the minimum degree 3 and bipartite graphs $G=(A, B, E)$ with even $|A|$ or $|B|$.
\end{abstract}

Keywords Edge weighting · Vertex coloring - 1-2-3 Conjecture

\footnotetext{
$凶$ Joanna Skowronek-Kaziów

J.Skowronek-Kaziow@wmie.uz.zgora.pl

1 University of Zielona Góra, Zielona Gora, Poland
} 


\section{Mathematics Subject Classification 05C15 - 05C85}

\section{Introduction}

In this paper we consider only non-trivial graphs, i.e., simple connected graphs with at least three vertices. A $k$-weighting of a graph is a mapping $w: E(G) \rightarrow\{1,2, \ldots, k\}$. We can define a vertex coloring $c(v)$ by $c(v)=\prod_{v \in e} w(e)$ for every $v \in V(G)$. A $k$-weighting of a graph $G$ is called multiplicative vertex-coloring if for every edge $u v$, $c(u) \neq c(v)$.

The study of edge weightings which are additive vertex-coloring, was initiated by Karoński et al. (2004). A $k$-weighting is additive vertex-coloring if for every edge $u v$, the sum of weights of the edges incident to $u$ is different than the sum of weights of the edges incident to $v$. They conjectured that every non-trivial graph permits an additive vertex-coloring 3-weighting (1-2-3 Conjecture), and proved this conjecture for 3colorable graphs. The best result concerning 1-2-3 Conjecture is given by Kalkowski et al. (2010), who presented an additive vertex-coloring 5-weighting for every nontrivial graph.

Different versions of vertex coloring from an edge $k$-weighting (by considering the sums, products, sequences, sets, or multisets of incident edge weights) were investigated by many authors in Addario-Berry et al. (2005), Bartnicki et al. (2009), Chang et al. (2011), Kalkowski et al. (2010), Karoński et al. (2004), Lu et al. (2011), Skowronek-Kaziów (2012), Stevens and Seamone (2013). Some authors distinguish all the vertices in a graph by their product colors (product irregularity strength of graphs, see Anholcer (2009, 2014), Darda and Hujdurovic (2014)). The total version of this problem was also investigated, where all the edges and vertices are weighted with integers, and the color of a vertex is the sum of its weight and the weights of the incident edges (see Przybyło and Woźniak (2010), Skowronek-Kaziów (2008)). In papers Czerwiński et al. (2009) and Grytczuk et al. (2013), the vertex version of vertex-coloring was presented, in this case, only the vertices are weighted, and the color of a vertex is the sum of integer weights of its neighbors. The problem of a proper edge weighting, such that no two neighbors are adjacent to the same set of weights, was also investigated, see Hocquard and Montassier (2013), Wang and Wang (2010), Wang (2007).

In 2011, Chang, Lu, Wu, Yu, and Zhang [Chang et al. (2011), Lu et al. (2011)] considered the graphs with additive vertex-coloring 2-weightings. In particular, they proved that 3-connected bipartite graphs, bipartite graphs with the minimum degree 1, and $r$-regular bipartite graphs with $r \geq 3$ permit an additive vertex-coloring 2weighting. In this paper, the multiplicative version of this problem is considered.

We fix the following notation. A graph $C_{n}$ and $P_{n}$ is a cycle and a path, respectively, with $n$ vertices. A graph $K_{m, n}$ is a complete bipartite graph. $N[v]=N(v) \cup\{v\}$ is the closed neighborhood of a vertex $v, d(v)$ is the degree of $v$, and $\delta(G)$ is the minimum degree of a vertex in a graph $G$. The graph $G=(A, B, E)$ is a bipartite graph with edge set $E$ and vertex bipartition $(A, B)$. 


\section{Graphs with multiplicative vertex-coloring 2-weightings}

The minimum $k$ for which $G$ has a multiplicative vertex-coloring $k$-weighting is denoted by $\mu(G)$. It is easy to see that $\mu(G) \geq 2$. The following question was asked in Skowronek-Kaziów (2012):

Question (2012, Skowronek-Kaziów (2012)): Is $\mu(G) \leq 3$ for every non-trivial graph $G$ ?

The question still remains unanswered, but the best result proved in SkowronekKaziów (2012) is $\mu(G) \leq 4$ for every non-trivial graph $G$. It was shown in SkowronekKaziów (2012) that every 3-colorable graph admits a multiplicative vertex-coloring 3-weighting. A natural problem to consider is whether every 2-colorable graph (i.e., a bipartite graph) has a multiplicative vertex-coloring 2-weighting. But the answer is no, since the cycle $C_{6}$ and the path $P_{6}$ do not admit a multiplicative vertex-coloring 2 -weighting. Notice that a non-trivial graph $G$ permits an additive vertex-coloring 1 -weighting if and only if $G$ has no adjacent vertices with the same degree, but there is no graph $G$ with $\mu(G)=1$. Of course, if $G$ has no adjacent vertices with the same degree, then $\mu(G)=2$.

Proposition 1 Let $G=(A, B, E)$ be a non-trivial connected bipartite graph and let $|A| \leq|B|$. Then $\mu(G)=2$ if there exists $v_{0} \in A$ such that $d\left(v_{0}\right)=|B|$.

Proof Let $G=(A, B, E)$ be a non-trivial bipartite graph with edge set $E$ and vertex bipartition $(A, B),|A| \leq|B|$. Let $v_{0} \in A$ such that $d\left(v_{0}\right)=|B|$. We can define a 2-weighting $w: E \rightarrow\{1,2\}$ of edges as follows: $w\left(v_{0} u\right)=2$ for all $u \in B$, and $w(e)=1$ for all remaining edges $e \in E$. Let $c(v)=\prod_{v \in e} w(e)$.

The above 2-weighting is multiplicative vertex-coloring, since $c\left(v_{0}\right)=2^{|B|} \geq 4$, $c(v)=1$ for $v \in A \backslash\left\{v_{0}\right\}$, and $c(u)=2$ for all $u \in B$.

From Proposition 1, $\mu\left(K_{m, n}\right)=2$.

Proposition 2 Let $G=(A, B, E)$ be a non-trivial connected bipartite graph. Then $\mu(G)=2$ if $|A|$ or $|B|$ is even.

Proof Let $G=(A, B, E)$ be a connected bipartite graph. Let $A=\left\{v_{1}, v_{2}, \ldots, v_{2 k}\right\}$, $k \geq 1$. For every $i \in\{1,2, \ldots, k\}$ choose a shortest $v_{i} \rightarrow v_{i+k}$ path $P_{i}$. Let $\left\{P_{1}, P_{2}, \ldots, P_{k}\right\}$ be the set of such chosen paths. Let $d_{i}(v)$ be the degree of a vertex $v$ in the path $P_{i}$ and let $\sigma(v)=\sum_{i=1}^{k} d_{i}(v)$ for every $v \in A \cup B$. Then $\sigma(v) \equiv 1(\bmod 2)$ for $v \in A$, and $\sigma(v) \equiv 0(\bmod 2)$ for $v \in B$. For every edge $e \in E$ let $a_{i}(e)=1$ if $e \in E\left(P_{i}\right)$, and $a_{i}(e)=0$ if $e \notin E\left(P_{i}\right)$. Put $\alpha(e)=\sum_{i=1}^{k} a_{i}(e)$. Now, let us define a 2-weighting $w: E \rightarrow\{1,2\}$ as follows: $w(e)=1$ if $\alpha(e) \equiv 0(\bmod 2)$, and $w(e)=2$ if $\alpha(e) \equiv 1(\bmod 2)$.

Then $c(v)=2^{2 s+1}$ if $v \in A$, and $c(u)=2^{2 s}$ if $u \in B$, for some $s \geq 0$.

Hence $c(v) \neq c(u)$ for every edge $v u \in E, v \in A, u \in B$. 
Corollary 3 Let $G$ be a non-trivial connected bipartite graph. Then $\mu(G)=2$ if $|V(G)|$ is odd.

Proposition 4 Let $G$ be a path or a cycle. Then $\mu(G)=2$ if and only if $G$ is a bipartite graph $G=(A, B, E)$ with even $|A|$ or $|B|$.

Proof Notice that every path $P_{2 k}$ (or a cycle $C_{2 k}$ ) with even $k \in N$ and every path $P_{n}$ with odd number $n$ of vertices is a bipartite graph $G=(A, B, E)$ with even $|A|$ or $|B|$.

So, let $G$ be a path $P_{2 k}$ (a cycle $C_{2 k}$ ) with odd $k \in N$ (which is a bipartite graph $G=$ ( $A, B, E$ ) with odd $|A|$ and odd $|B|$ ) or a cycle $C_{n}$ with odd $n$ (which is not bipartite). Assume that there is a 2-weighting of $G$ which is multiplicative vertex-coloring. Then, the weights should appear on consecutive edges as follows: 2,2,1,1,2,2,1,1,2,2,... But then there are two adjacent vertices $u$ and $v$ with the same product colors $c(u)=c(v)$, a contradiction.

Proposition 5 Let $G$ be a non-trivial connected bipartite graph. Then $\mu(G)=2$ if there exists a vertex with at least two neighbours of degree 1.

Proof Let $G=(A, B, E)$ be a connected bipartite graph. By Proposition 2, we can assume that both $|A|$ and $|B|$ are odd. Assume that $d(x)=1$ for some vertex $x$ in $A$, where $x$ is adjacent to $v \in B$ and $v$ is adjacent to $y \in A, d(y)=1, x \neq y$. Then $G-x=(A \backslash\{x\}, B, E \backslash\{x v\})$ is a bipartite graph with $|A \backslash\{x\}|$ even. From the proof of Proposition 2, a graph $G-x$ has a multiplicative vertex-coloring 2-weighting $w$ such that $w(y v)=2$. Extending this 2-weighting to $G$ by assigning $w(x v)=1$, we get a multiplicative vertex-coloring 2-weighting of $G$.

Proposition 6 Let $G$ be a non-trivial connected bipartite graph. Then $\mu(G)=2$ if there exists a vertex $x$ such that $d(x) \geq 3$ and a graph $G-N[x]-\{v: v$ is isolated in $G-N[x]\}$ is connected.

Proof Let $G=(A, B, E)$ be a nontrivial connected bipartite graph with a vertex $x \in B$ such that $d(x) \geq 3$ and $G_{1}=G-N[x]-\{v: v$ is isolated in $G-N[x]\}$ is connected. By Proposition 2, we may assume that both $|A|$ and $|B|$ are odd. Let $G_{1}=\left(A_{1}, B_{1}, E_{1}\right)$, where $A_{1}=A \backslash N(x)$.

Case $1 d(x)$ is odd. In this case $\left|A_{1}\right|$ is even. It follows from the proof of Proposition 2 that $G_{1}$ has a 2-weighting $w$ such that $c(v)=2^{2 s+1}$ if $v \in A_{1}$, and $c(v)=2^{2 s}$ if $v \in B_{1}$, for some $s \geq 0$. We can extend this 2-weighting to $G$ by assigning weight 2 to each edge $x u, u \in N(x)$, and weight 1 to the remaining edges. Then it is multiplicative vertex-coloring, since $c(v)=2^{2 s}$ for $s \geq 0$ and $v \in B \backslash\{x\}, c(x)=2^{d(x)} \geq 8$, $c(u)=2$ for $u \in N(x)$.

Case $2 d(x)$ is even. In this case $\left|A_{1}\right|$ is odd, and there is a vertex $u_{0} \in N(x)$ adjacent to a vertex $v_{0} \in B_{1}$. Let $G$ ' be the graph obtained from $G_{1}$ by adding the vertex $u_{0}$ and the edge $u_{0} v_{0}$. It follows from the proof of Proposition 2 that $G^{\prime}$ has a 2-weighting $w$ such that $c(v)=2^{2 s+1}$ if $v \in A_{1} \cup\left\{u_{0}\right\}$, and $c(v)=2^{s}$ if $v \in B_{1}, s \geq 0$. Notice that $w\left(u_{0} v_{0}\right)=2$. We can extend this 2 -weighting to $G$ by assigning weight 2 to each edge $x u, u \in N(x) \backslash\left\{u_{0}\right\}$, and weight 1 to the remaining edges. Then it is multiplicative vertex-coloring, and $c(v)=2^{2 s}$ for $s \geq 0$ and $v \in B-\{x\}, c(x)=2^{d(x)-1} \geq 8$, $c(u)=2$ for $u \in N(x)$. 
Corollary 7 Let $G$ be a non-trivial connected bipartite graph. Then $\mu(G)=2$ if $\delta(G) \geq 3$.

Proof Let $G=(A, B, E)$ be a connected bipartite graph with $\delta(G) \geq 3$. By Proposition 2, we may assume that both $|A|$ and $|B|$ are odd. Choose a vertex $x \in B$ such that the size of the maximum component $G_{1}=\left(A_{1}, B_{1}, E_{1}\right), A_{1} \subseteq A \backslash N(x)$, of $G-N[x]$ is as large as possible. Let $G^{\prime}=\left(A^{\prime}, B^{\prime}, E^{\prime}\right)$ be another component of $G-N[x]$, and let $x^{\prime} \in B^{\prime}$. Then $G_{1} \cup N[x]$ is contained in a component of $G-N\left[x^{\prime}\right]$ which is larger than $G_{1}$ and which contradicts our choice of $x$. Therefore, all other components of $G-N[x]$ (except $G_{1}$ ) are isolated vertices in $B$, and the thesis follows from Proposition 6.

From above, all $r$-regular bipartite graphs, $r \geq 2$, (except cycles of length $2 k, k$ is odd) permit a multiplicative vertex-coloring 2 -weighting.

Proposition 8 Let $G$ be a non-trivial connected bipartite graph, and let $x \in V(G)$ be at distance 2 from some vertex of degree 1 . Then $\mu(G)=2$ if a graph $G-x-\{v: v$ is isolated in $G-x\}$ is connected.

Proof By Proposition 2, we may assume that both $|A|$ and $|B|$ are odd. Let $x \in A$ be a vertex of a path $x, u_{1}, v_{1}$ of length 2 where $d\left(v_{1}\right)=1$. Now, let $G=(A, B, E)$ and let $G^{\prime}=G-x-\{v: v$ is isolated in $G-x\}$ is connected.

It follows from the proof of Proposition 2 that $G^{\prime}$ has a multiplicative vertexcoloring 2-weighting $w$ such that $w\left(u_{1} v_{1}\right)=2$ and $4 \leq c\left(u_{1}\right)=2^{2 s}, s \geq 1$. Now, we can extend this 2 -weighting to $G$ by changing the weight of $u_{1} v_{1}$ from 2 to 1 , assigning $w\left(x u_{1}\right)=2$ and $w(e)=1$ to all other edges $e$ in $G$. In this way we get a multiplicative vertex-coloring 2-weighting of $G$ with $c(x)=2, c\left(u_{1}\right) \geq 4, c\left(v_{1}\right)=1$ and $c(u)=2^{2 s}, s \geq 0$, for all $u \in N(x)$.

Every tree permits an additive vertex-coloring 2-weighting (see Lu et al. (2011)) but not always a multiplicative vertex-coloring 2-weighting (the good example is the path $P_{2 k}$, with $\mu\left(P_{2 k}\right)=3$ for odd $\left.k\right)$.

It is a known fact that if $T$ is a tree in which every vertex has a degree 1 or 3 , then there is a vertex adjacent to two leaves.

Hence we get several sufficient conditions for a tree $T$ with $\mu(T)=2$ :

Corollary 9 Let $T$ be a tree. Then $\mu(T)=2$, if at least one of the following conditions holds:

a) Thas a vertex adjacent to two leaves;

b) every vertex in $T$ has a degree 1 or 3;

c) Thas a vertex $x$ which is at distance 2 from a leaf and $T-x-\{v: v$ is isolated in $T-x$ \} is connected;

d) $T$ has an odd number of vertices.

We can conclude the paper by the following problem:

Problem Classify all bipartite graphs (in particular trees) which permit a multiplicative vertex-coloring 2-weighting. 
Open Access This article is distributed under the terms of the Creative Commons Attribution 4.0 International License (http://creativecommons.org/licenses/by/4.0/), which permits unrestricted use, distribution, and reproduction in any medium, provided you give appropriate credit to the original author(s) and the source, provide a link to the Creative Commons license, and indicate if changes were made.

\section{References}

Addario-Berry L, Alred REL, Dalal K, Reed BA (2005) Vertex colouring edge partitions. J Combin Theory Ser B 94:237-244

Anholcer M (2009) Product irregularity strength of graphs. Discret Math 309:6434

Anholcer M (2014) Product irregularity strength of certain graphs. ARS Math Contemp 7:23-29

Bartnicki T, Grytczuk J, Niwczyk S (2009) Weight choosability of graphs. J Graph Theory 60(3):242-256

Chang GJ, Lu C, Wu J, Yu QL (2011) Vertex-coloring edge-weightings of graphs. Taiwan J Math 15(4):1807-1813

Czerwiński S, Grytczuk J, Żelazny W (2009) Lucky labelings of graphs. Inf Process Lett 109:1078-1081

Darda R, Hujdurovic A (2014) On bounds for the product irregularity strength of graphs. Graphs Combin 31:1347-1357

Grytczuk J, Bartnicki T, Czerwiński S, Bosek B, Matecki G, Żelazny W (2013) Additive colorings of planar graphs. Graphs Combin 30:1-12

Hocquard H, Montassier M (2013) Adjacent vertex-distinguishing edge coloring of graphs with maximum degree $\Delta$. J Combin Optim 26(1):152-160

Kalkowski M, Karoński M, Pfender F (2010) Vertex-coloring edge-weighting: toward the 1-2-3-conjecture. J Combin Theory Ser B 100:347-349

Karoński M, Łuczak T, Thomason A (2004) Edge weights and vertex colours. J Combin Theory Ser B 91:151-157

Lu H, Yu Q, Zhang Cun-Quan (2011) Vertex-coloring 2-edge-weighting of graphs. Eur J Combin 32:21-27

Przybyło J, Woźniak M (2010) On a 1,2 conjecture. Discret Math Theor Comput Sci 12(1):101-108

Skowronek-Kaziów J (2008) 1, 2 conjecture-the multiplicative version. Inf Process Lett 107(3-4):93-95

Skowronek-Kaziów J (2012) Multiplicative vertex-colouring weightings of graphs. Inf Process Lett 112:191-194

Stevens B, Seamone B (2013) Sequence variations of the 1-2-3 conjecture and irregularity strength. Discret Math Theor Comput Sci 15(1):15-28

Wang W, Wang Y (2010) Adjacent vertex distinguishing edge-colorings of graphs with smaller maximum average degree. J Combin Optim 19:471-485

Wang Haiying (2007) On the adjacent vertex-distinguishing total chromatic numbers of the graphs with $\Delta(G)=3$. J Combin Optim 14(1):87-109 